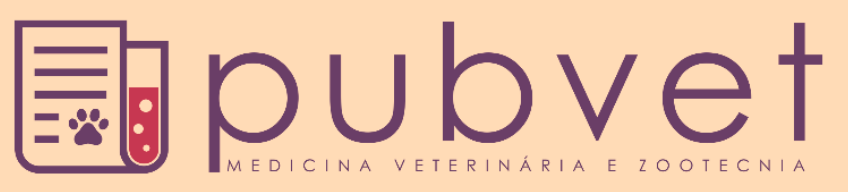

ISSN $1982-1263$

https://doi.org/10.31533/pubvet.v15n11a968.1-6

\title{
Técnica de rinoplastia com uso de bisturi ultrassônico em felino da raça Persa
}

\author{
Flavia Alves Gomes ${ }^{1 *} \bullet$, Ivan Torres Gregório da Silva ${ }^{\bullet}$, Debora Rodrigues Ferreira ${ }^{0}$, \\ Thainá Elis Fonseca de Aquino ${ }^{3}$
}

IDiscente em Medicina Veterinária pela Universidade Federal Fluminense, Rio de Janeiro, Brasil.

${ }^{2}$ Graduado em Medicina Veterinária pela Universidade Federal Fluminense, pós-graduado em Cirurgia de Tecidos Moles pela Anclivepa-SP, Rio de Janeiro, Brasil.

${ }^{3}$ Discente de Medicina Veterinária pelo Centro Universitário Univeritas, São Gonçalo, Brasil.

*Autor para correspondência, E-mail: flavia_alves@id.uff.br

\begin{abstract}
Resumo. A estenose de narina é considerada uma alteração primaria na síndrome braquicefálica, que tem como característica uma abertura nasal estreita limitando a passagem do ar, sendo facilmente observada externamente. Cães e gatos que demonstram narina estenosada apresentam grande esforço respiratório, cansaço, dispneia, sono inquieto e roncos. O tratamento é a correção cirúrgica por meio do procedimento de rinoplastia, tendo em vista que a síndrome braquicefálica é progressiva, o prognostico se torna favorável quando a cirurgia é feita em animais em que a síndrome não esteja tão avançada. $\mathrm{O}$ presente relato tem objetivo apresentar um gato da raça persa, de nove anos de idade com histórico de intolerância ao exercício e secreção nasal frequente. $\mathrm{O}$ animal foi submetido à técnica de rinoplastia com o uso do bisturi ultrassônico, o que permitiu uma recuperação cirúrgica mais rápida e pós cirúrgico imediato já apresentando melhora nos sinais clínicos.
\end{abstract}

Palavras chaves: Braquicefálicos, estenose de narina, raça persa

\section{Rhinoplasty technique using ultrasonic scalpel in Persian feline}

\begin{abstract}
The nostril stenosis is considered a primary alteration in brachycephalic syndrome, which features a narrow nasal opening limiting the air passage, being easy to be externally noted. Dogs and cats that show stenosed nostrils have great respiratory effort, tiredness, and snoring. The treatment is surgical correction through the rhinoplasty procedure. Considering that the brachycephalic syndrome is progressive, the prognosis becomes favorable when animals are taken under surgery when the syndrome has not advanced so much. The present report aims to present a nine-year-old Persian cat with a history of intolerance to exercises and frequent nasal discharge. A the animal was submitted to the rhinoplasty procedure with the use of the ultrasonic scalpel, which led to a faster surgical recovery and immediate post-surgical, already showing clinical signs of improvement.
\end{abstract}

Keywords: Brachycephalic, nostril stenosis, persian

\section{Introdução}

A síndrome braquicefálica apesar de ser mais comum em cães, mas gatos como os persa e himalaio também podem ser acometidos (Teichmann et al., 2012). Se caracterizando por alterações anatômicas no trato respiratório superior. Animais acometidos apresentam como alteração primaria estenose de narina e prolongamento do palato mole, podendo apresentar as duas ou apenas uma das alterações. 
Devido o esforço respiratório, é possível observar eversão dos sáculos laríngeos e colapso de brônquio em animais que apresentam a síndrome mais avançada. $O$ tratamento das alterações primaria amenizam as alterações secundaria, sendo recomendada uma reavaliação após o tratamento cirúrgico (Riggs et al., 2019). Os sinais clínicos geralmente relatados são respiração barulhenta, intolerância ao exercício, sono inquieto e cianose. Alguns animais apresentam sinais gastrointestinais concomitantes (Fossum, 2014).

A estenose dos orifícios nasais acomete cerca de 48\% dos casos (Jericó et al., 2015) e se caracteriza pela malformação congênitas das cartilagens nasais (Figura 1). Exerce-se uma pressão negativa cada vez maior para respirar gerando pressão intratraqueal e intrafaringeana. O diagnostico se dá pelo exame físico, a estenose de narina é de fácil visualização, durante a inspiração as narinas podem ser puxadas medialmente ou se manter relativamente estacionária, em vez de se abrirem (Fossum, 2014). Além de prejudicar a respiração, a estenose de narina também influencia na termorregulação.
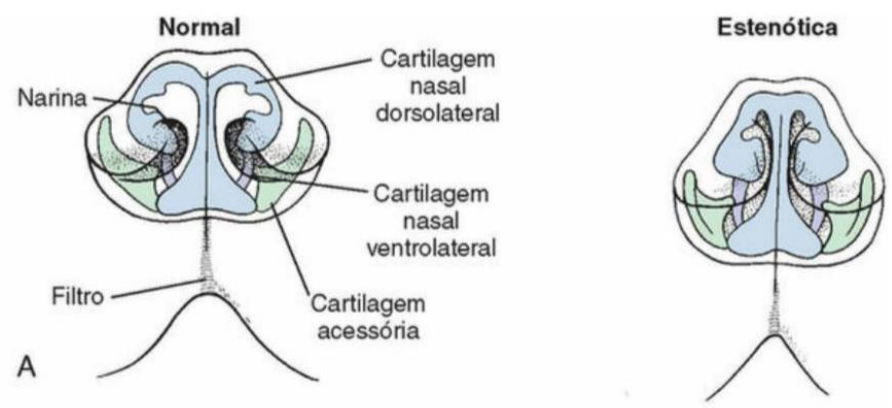

Figura 1. Aparência normal e estenótica das narinas (Fossum, 2014)

Eletrocardiograma e ecocardiograma são necessários para a investigação de doenças secundarias. O prognostico do animal submetido à cirurgia em sua maioria é favorável (Nelson \& Couto, 2015).

Este trabalho relata o caso de um gato, da raça persa diagnosticado com síndrome braquicefálica apresentando narina estenosada, levando em consideração diagnostico, técnica cirúrgica, plano anestésico e prognostico.

\section{Relato de caso}

Um gato da raça persa, de nove anos pesando $4,0 \mathrm{~kg}$, atendido com a queixa de intolerância ao exercício e secreção nasal frequente. $\mathrm{Na}$ anamnese foi diagnosticada síndrome braquicefálica em grau II de acordo com a escala Brachycephalic obstructive airway syndrome (BOAS), tendo o zero como um cão que não apresenta nenhum sinal e o grau três para o caso mais severo (Riggs et al., 2019).

Constituindo grande esforço respiratório devido à estreita abertura nasal, o animal não apresentava prolongamento do palato mole (confirmado no ato anestésico). Foi realizado exame de hemograma (Anexo 1), exames bioquímicos (Anexo 2) e exame cardiológico (Figuras 2 e 3 ), onde foi constatado cardiomiopatia hipertrófica leve, é a afecção cardíaca mais comum nessa raça (Ware, 2014).

Após os exames físico, laboratoriais e de imagem foi decidido pela técnica cirúrgica de rinoplastia para o paciente, onde foi feita a ressecção das narinas estenóticas para uma abertura permanente das narinas externas. O procedimento foi feito com o animal em decúbito esternal, a antissepsia no plano nasal foi feita com clorexidina degermante e alcoólico. Na medicação pré-anestésica foi feito $1 \mathrm{mg} / \mathrm{kg}$ ketamina, $5 \mathrm{mcg} / \mathrm{kg}$ dexmedetomidina, $0,15 \mathrm{mg} / \mathrm{kg}$ de midazolam, $0,3 \mathrm{mg} / \mathrm{kg}$ de metadona, indução com propofol, anestesia com isoflurano e infusão de fetanil. Animais com a síndrome braquicefálica carecem de maior cuidado durante o ato anestésico, tendo como preocupação desenvolvimento de obstruções das vias aéreas, completa ou imparcial, e predisposição para bradicardia e regurgitação (Tranquilli et al., 2013).

O procedimento de rinoplastia foi feito com o uso do bisturi ultrassônico, que permite incisões homeostáticas com baixo dano térmico aos tecidos adjacentes, o aquecimento secundário faz com que as proteínas desnaturadas formem um coágulo. O tecido é cortado e cauterizado simultaneamente. As margens das narinas foram presas com uma pinça de Brown Adson, mantendo-a presa, foi feita uma ressecção da borda vertical com o uso do bisturi ultrassónico, dispensando o uso de suturas. 


\begin{tabular}{|c|c|}
\hline Medidas & \\
\hline \begin{tabular}{|l} 
2D \\
Diâm AE \\
Diâmetro Raiz Ao \\
LA/Ao \\
Ao/LA
\end{tabular} & $\begin{array}{l}0.93 \mathrm{~cm} \\
0.69 \mathrm{~cm} \\
1.36 \\
0.74\end{array}$ \\
\hline Mado-M e PW & \\
\hline sivd & $0.55 \mathrm{~cm}$ \\
\hline DIVEd & $1.30 \mathrm{~cm}$ \\
\hline PPVEd & $0.47 \mathrm{~cm}$ \\
\hline SIVs & $0.79 \mathrm{~cm}$ \\
\hline DIVEs & $0.74 \mathrm{~cm}$ \\
\hline VDF(Teich) & $\begin{array}{l}0.62 \mathrm{~cm} \\
4.18 \mathrm{ml}\end{array}$ \\
\hline VSF(Teich) & $0.92 \mathrm{ml}$ \\
\hline FE(Teich) & $77.97 \%$ \\
\hline \%Delta D & $42.80 \%$ \\
\hline SV(Teich) & $3.26 \mathrm{ml}$ \\
\hline $\begin{array}{l}\text { Tempo } \\
\text { FC }\end{array}$ & $\begin{array}{l}323.33 \mathrm{~ms} \\
185.57 \mathrm{BPM}\end{array}$ \\
\hline $\mathrm{DC}($ Teich $)$ & $0.57 \mathrm{l} / \mathrm{min}$ \\
\hline Veloc. E VM & $0.82 \mathrm{~m} / \mathrm{s}$ \\
\hline T. desac. VM & $36.25 \mathrm{~ms}$ \\
\hline Rampa Des VM & $22.63 \mathrm{~m} / \mathrm{s}^{2}$ \\
\hline Velocid. A VM & $0.01 \mathrm{~m} / \mathrm{s}$ \\
\hline $\begin{array}{l}\text { Relação E/A VM } \\
\text { PHT VM }\end{array}$ & $\begin{array}{l}87.00 \\
10.51 \mathrm{~ms}\end{array}$ \\
\hline$A \vee M$ & $20.93 \mathrm{~cm}^{2}$ \\
\hline IVRT & $30.00 \mathrm{~ms}$ \\
\hline $\begin{array}{l}\text { Vmáx VP } \\
\text { Grad. máx VP }\end{array}$ & $\begin{array}{l}0.67 \mathrm{~m} / \mathrm{s} \\
1.79 \mathrm{mmHg}\end{array}$ \\
\hline
\end{tabular}

Figura 2. Medidas do exame cardiológico de gato persa com idade de nove anos
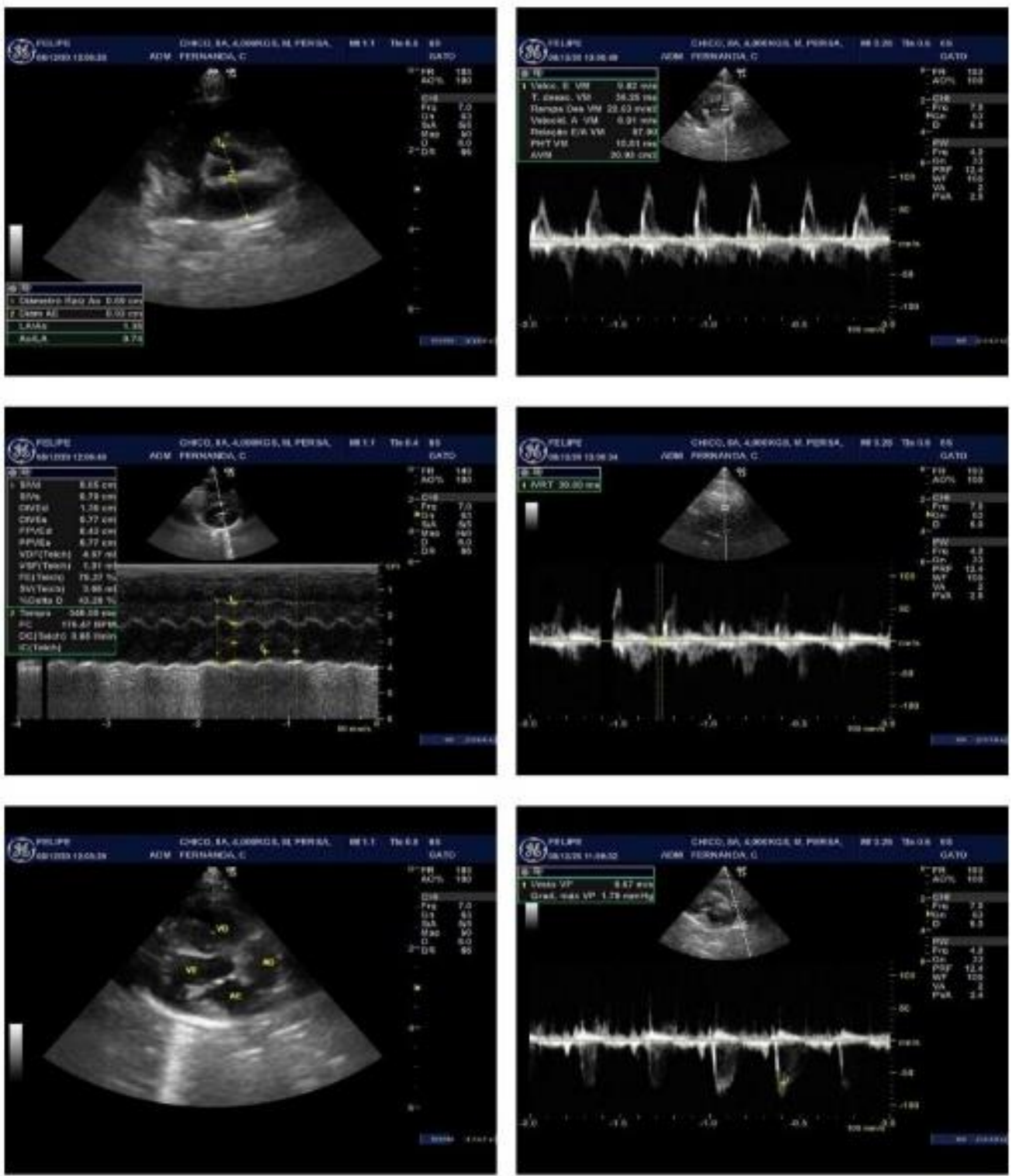

Figura 3. Exame cardiológico do gato da raça persa com idade de nove anos 
Medicamentos prescritos no pós-operatório foram cefradroxila $25 \mathrm{mg} / \mathrm{kg}$ uma vez ao dia por dez dias, dipirona gotas $5 \mathrm{mg} / \mathrm{kg}$ de $12 \mathrm{em} 12$ horas, prednisolona $1 \mathrm{mg} / \mathrm{kg} 12 \mathrm{em} 12$ horas por três dias e $0,5 \mathrm{mg} / \mathrm{kg}$ de $12 \mathrm{em} 12$ horas por mais três dias, gaviz $0,5 \mathrm{mg} / \mathrm{kg} 12 \mathrm{em} 12$ horas, rutina $30 \mathrm{mg} / \mathrm{kg}$ de 8 em 8 horas.

\section{Discussão e resultados}

O tratamento cirúrgico é o mais indicado para correção das narinas estenóticas, sendo o único tratamento definitivo (Nelson \& Couto, 2015). A maioria dos animais submetidos à cirurgia de correção apresenta uma boa recuperação (Lameu et al., 2020). O uso do bisturi ultrassônico foi o diferencial durante a cirurgia, oferecendo baixo dano a tecidos adjacentes, com uma cirurgia mais segura, sem suturas e um pós-cirúrgico com boa recuperação. $\mathrm{O}$ animal não apresentou edema, sangramento, inapetência e dor.

Após o animal ser extubado, acordou sem complicações e com sinais de melhoras já no pós-cirúrgico imediato. Após a cirurgia o animal já foi para casa, tendo em vista que o uso do bisturi ultrassônico diminui o risco de edemas, principal complicação neste tipo de procedimento.

Com a cirurgia, o animal demonstrou melhora considerável em seu quadro, apresentando melhor qualidade de vida, respirando sem esforço, tornando-se tolerável ao exercício, sono mais tranquilo, mais ativo e sem secreção nasal constante, apesar da idade mais avançada, apresentou uma melhora satisfatória da síndrome braquicefalica.

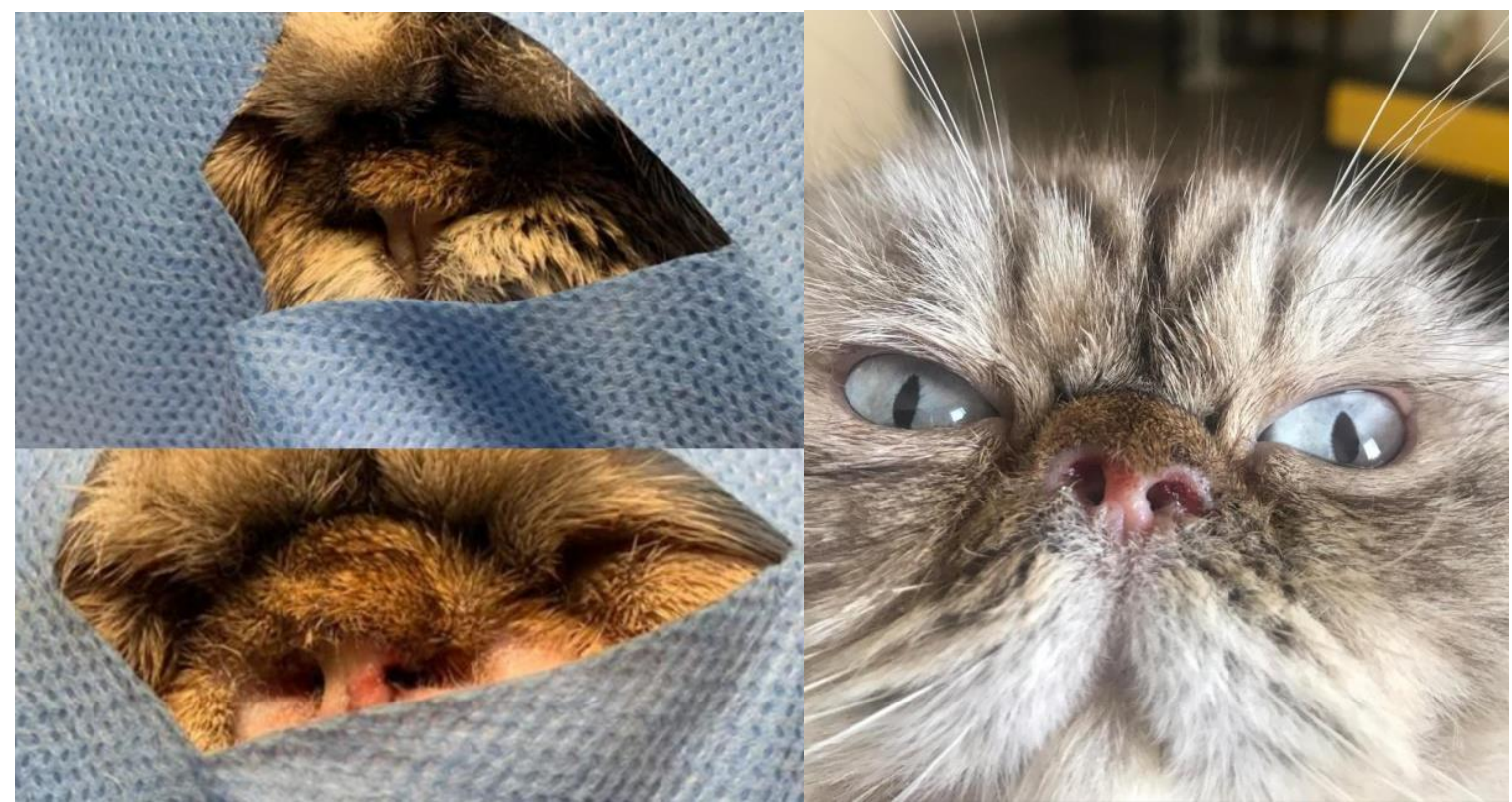

Figura 6. Antes e depois do procedimento de rinoplastia com Figura 7. Recuperação do animal após 15 dias do o uso do bisturi ultrassónico. procedimento.

\section{Conclusão}

A rinoplastia é único tratamento definitivo, que se tornou de total importância para melhora da qualidade de vida do animal e evitando alterações secundarias da síndrome braquicefalica. A interferência cirúrgica precoce favorece um melhor prognostico. $\mathrm{O}$ animal demonstrou melhora clinica já no pós-cirúrgico imediato, após a recuperação completa o paciente demonstrou melhora no quadro respiratório.

\section{Referência bibliográfica}

Fossum, T. W. (2014). Cirurgia de pequenos animais (4th ed., Vol. 1). Elsevier Brasil.

Jericó, M. M., Kogika, M. M., \& Andrade Neto, J. P. (2015). Tratado de medicina interna de cães e gatos. Guanabara Koogan. 
Lameu, G. R., Silva, P. Í. B., Menezes, A. D. R., Alves, C. C., Soares, M. A., Bilhalva, M. A., Evaristo, T. A., Pellegrin, T. G., Vasconcellos, A. L., \& Costa, P. P. C. (2020). Síndrome braquicefálica em cães: Revisão. PUBVET, 14(10), 1-7.

Nelson, R. W., \& Couto, C. G. (2015). Medicina interna de pequenos animais (Issue 1). Elsevier Editora.

Riggs, J., Liu, N., Sutton, D. R., Sargan, D., \& Ladlow, J. F. (2019). Validation of exercise testing and laryngeal auscultation for grading brachycephalic obstructive airway syndrome in pugs, French bulldogs, and English bulldogs by using whole-body barometric plethysmography. Veterinary Surgery, 48(4), 488-496.

Teichmann, C., Pereira, M. A. M., \& Reimann, P. (2012). Alterações anatômicas em cães com síndrome braquicefálica. UNICRUZ.

Tranquilli, W. J., Thurmon, J. C., \& Grimm, K. A. (2013). Lumb and Jones'veterinary anesthesia and analgesia. John Wiley \& Sons.

Ware, W. A. (2014). Doenças miocárdicas do gato. In R. E. Nelson \& C. G. Couto (Eds.), Medicina interna de pequenos animais (pp. 145-158).

Histórico do artigo:

Recebido: 2 de julho de 2021

Aprovado: 8 de agosto de 2021
Licenciamento: Este artigo é publicado na modalidade Acesso Aberto sob a licença Creative Commons Atribuição 4.0 (CC-BY 4.0), a qual permite uso irrestrito, distribuição, reprodução em qualquer meio, desde que o autor $\mathrm{e}$ a fonte sejam devidamente creditados.

Anexo 1. Exame de hemograma do gato da raça persa com idade de nove anos

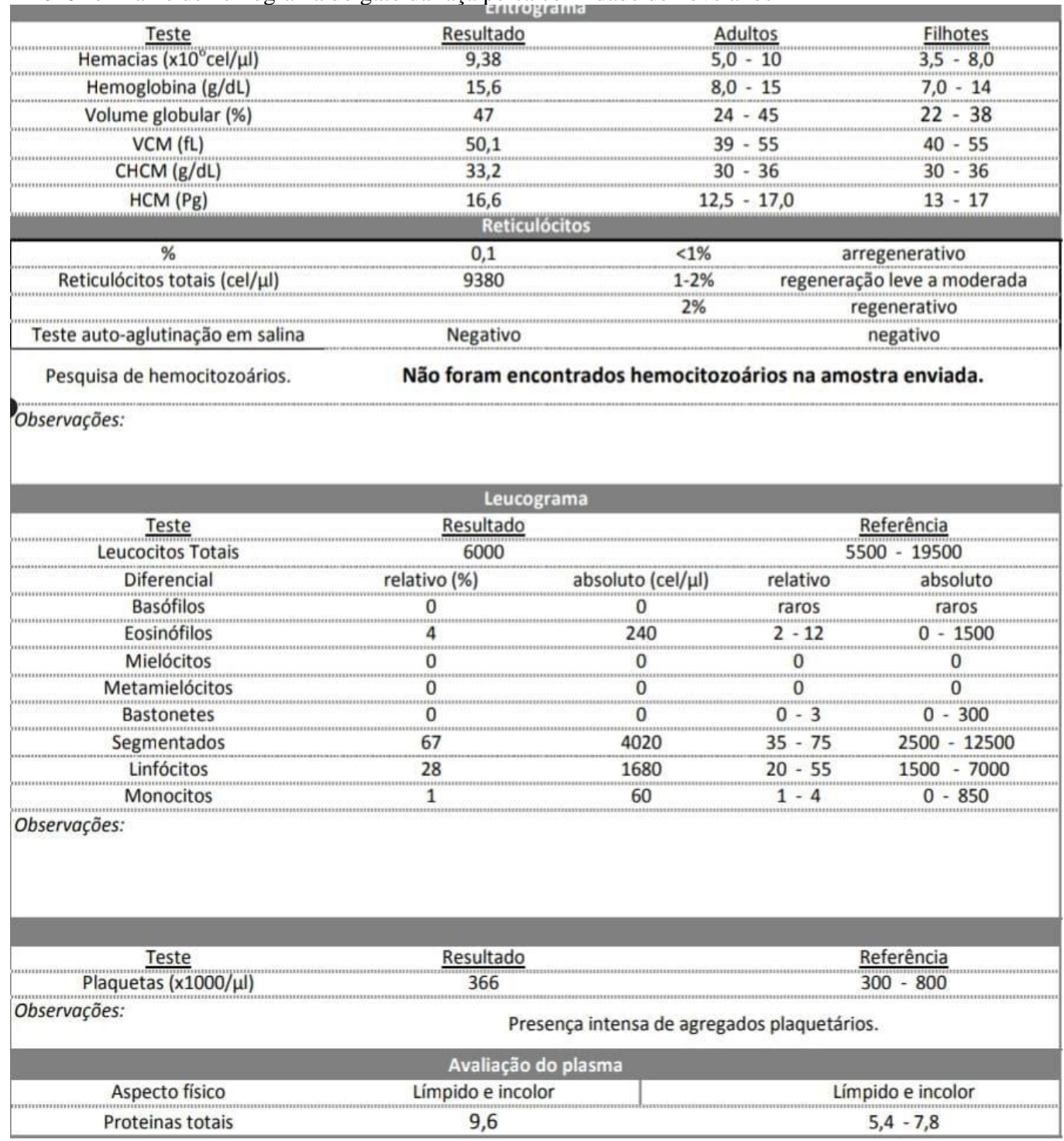


Anexo 2. Exame de bioquímica do gato da raça persa com idade de nove anos

\begin{tabular}{|cccc|}
\hline Analito & Resultado & \multicolumn{2}{c|}{ Referência } \\
\hline & & caninos & felinos \\
\hline ALT (U/L) & 77 & $20-98$ & $35-175$ \\
\hline Bilirrubina total (mg/dL) & 0,02 & $0,0-0,2$ & $0,0-0,2$ \\
\hline Bilirrubina direta (mg/dL) & 0,01 & $0,0-0,1$ & $0,0-0,1$ \\
\hline Bilirrubina indireta (mg/dL) & 0,01 & $0,0-0,2$ & $0,0-0,2$ \\
\hline Creatinina (mg/dL) & 0,74 & $0,5-1,5$ & $0,8-1,5$ \\
\hline Fosfatase alcalina (U/L) & 114 & $17-111$ & $13-83$ \\
\hline GGT (U/L) & 0,7 & $0,0-6,0$ & $0,0-2,0$ \\
\hline Proteína Total (g/dL) & 5,58 & $5,3-7,0$ & $6,3-8,3$ \\
\hline Albumina (g/dL) & 3,15 & $3,1-4,2$ & $2,8-4,2$ \\
\hline Globulina (g/dL) & 2,43 & $1,9-3,6$ & $2,7-4,9$ \\
\hline Relação A:G & 1,30 & $0,9-2,1$ & $0,6-1,6$ \\
\hline Uréia (mg/dL) & 33 & $10-60$ & $20-60$ \\
\hline Observações: & & \\
\hline & & \\
\hline
\end{tabular}

